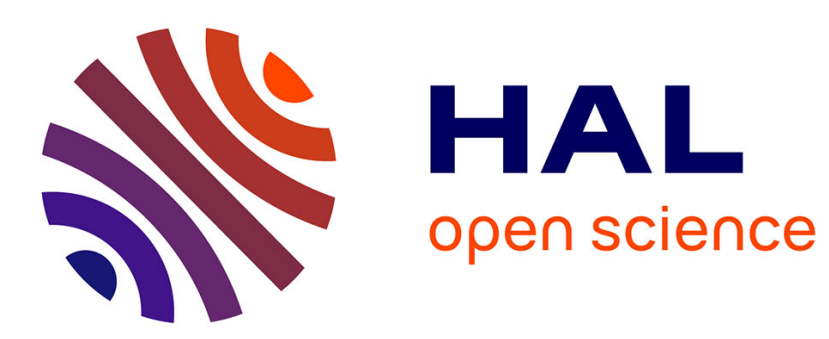

\title{
Invariants of Six Points and Projective Reconstruction from Three Uncalibrated Images \\ Long Quan
}

\section{To cite this version:}

Long Quan. Invariants of Six Points and Projective Reconstruction from Three Uncalibrated Images. IEEE Transactions on Pattern Analysis and Machine Intelligence, 1995, 17 (1), pp.34-46. 10.1109/34.368154 . inria-00590047

\section{HAL Id: inria-00590047 https://hal.inria.fr/inria-00590047}

Submitted on 3 May 2011

HAL is a multi-disciplinary open access archive for the deposit and dissemination of scientific research documents, whether they are published or not. The documents may come from teaching and research institutions in France or abroad, or from public or private research centers.
L'archive ouverte pluridisciplinaire HAL, est destinée au dépôt et à la diffusion de documents scientifiques de niveau recherche, publiés ou non, émanant des établissements d'enseignement et de recherche français ou étrangers, des laboratoires publics ou privés. 


\title{
Invariants of Six Points and Projective Reconstruction from Three Uncalibrated Images
}

\author{
Long QUAN
}

Abstract-There are three projective invariants of a set of six points in general position in space. It is well known that these invariants cannot be recovered from one image, however an invariant relationship does exist between space invariants and image invariants. This invariant relationship is first derived for a single image. Then this invariant relationship is used to derive the space invariants, when multiple images are available.

This paper establishes that the minimum number of images for computing these invariants is three, and the computation of invariants of six points from three images can have as many as three solutions. Algorithms are presented for computing these invariants in closed form.

The accuracy and stability with respect to image noise, selection of the triplets of images and distance between viewing positions are studied both through real and simulated images.

Applications of these invariants are also presented. Both the results of Faugeras [1] and Hartley et al. [2] for projective reconstruction and Sturm's method [3] for epipolar geometry determination from two uncalibrated images with at least seven points are extended to the case of three uncalibrated images with only six points.

Keywords - invariant, projective reconstruction, epipolar geometry, uncalibrated images, projective geometry, self-calibration.

\section{IntRoduCtion}

Geometric invariants are playing a more and more important role in machine vision applications. A lot of work for recognition and shape description using invariants has already been reported, for instance $c f$. the collection book [4] and [5], [6], [1], [7], [8], [9], [10], [2], [11], [12]. Most of the invariants [13], [14] are derived for planar objects using geometric entities such as points, lines and conics, since in this case, there exists a plane projective transformation between object and image space. Plane projective geometry provides an ideal mathematical tool for describing this. As for general geometric configurations in space, it has been shown that it is not possible to estimate invariants from a single image [15], except for some constraint geometric configurations [16]. Therefore, one (cf. [17], [1], [2], [11], [18]) basically deals with space projective invariants from two images, provided that the epipolar geometry, or the fundamental matrix [19], [20] of the two images is determined $a$ priori.

This paper is concerned with the computation of the invariants of sets of six points in space from three images taken with uncalibrated cameras, assuming that the correspondences between image points are known. The main new results obtained in this paper are that the minimum number of images for computing the invariants of six points is three; and the computation of invariants from three images can have as many as three solutions. All solutions

Long Quan is with LIFIA-CNRS-INRIA, 46, avenue Felix Viallet, 38031 Grenoble, France. E-mail: Long.Quan@imag.fr. are given in closed form. As a consequence of these results, both Sturm's method for epipolar geometry determination and projective reconstruction of Faugeras [1] and Hartley et al. [2] developed for two uncalibrated images is extended to the case of three uncalibrated images. Interestingly, in both two-camera and three-camera cases, the maximum number of solutions is three from the minimal data, i.e. six points for three images and seven points for two images. Also, with the help of such results, it may be established that the method of camera self-calibration proposed by Maybank and Faugeras [21] can be achieved with only six points of three images taken by the camera. Part of this work was also presented in [22].

Barrett et al. [17] have considered invariants from multiple images in order to solve the "transfer problem" in photogrammetry. Faugeras [1] and Hartley et al. [2] have shown that a set of points in 3D can be reconstructed up to a collineation from the point correspondences of two images taken with uncalibrated cameras. Similar results are presented by Mohr et al. in [6] for general multiple uncalibrated images using numerical minimization techniques. This permits the computation of projective invariants for sets of points visible from two or more images. In [6], [11], it is mentioned that it might be possible to get invariants from three images, however no explicit solutions are obtained. For other sets of geometric entities, invariants are obtained from two images and some experimental results are presented in [9], [11], [18]. However all these approaches using only two images are essentially based on the a priori determination of the epipolar geometry of the two images. The epipolar geometry may be algebraically determined, up to three solutions, with a minimum of seven points by Sturm's method [3], [19] or other equivalent algebraic methods based on the matrix representation of the epipolar geometry [23], [19]. However, Sturm's method is numerically unstable [20]. When more than eight points are available, numerical minimization methods are used to determine it. Therefore, to compute the invariants of six points, the correspondences of more than six points are needed for two images.

In comparison with related work, the method proposed in this paper does not need to first estimate the epipolar geometry between cameras, only the correspondences of six points are necessary, however a third image is needed to get algebraic solutions.

The development of this work is largely inspired by the old mathematical work on invariants of Coble [24] and the more recent work of Faugeras [1]. We assume that readers are familiar with elementary projective geometry and invariant theory, which can be found in [25], [24], [26], [27].

The paper is organized as follows. In Section II, the invari- 
ants of the set of six points in space are briefly reviewed. Then in Section III we will show that although invariants of a general set of points can not be obtained from one image, there exists a simple invariant relationship between the invariants of the set of 6 points in space and the invariants of their projected points in an image. Later in Section IV, this invariant relationship for one image will be applied to three images to compute invariants in space. Applications of the computation of invariants for projective reconstruction, epipolar geometry determination and self-calibration are discussed in Section V. Experimental results for the stability of these invariants and projective reconstruction are presented in Section VI. Future directions are discussed in Section VII.

\section{REVIEW OF INVARIANTS OF SIX POINTS IN SPACE}

Given a set of points or other kind of geometric configurations, the number of invariants is, roughly speaking, the difference between the dimension of the configuration and the dimension of the transformation group that acts on the configuration, if the dimension of the isotropy group of the configuration is null ( $c f$. [9], [4]). For a set of 6 points in $\mathcal{P}^{3}$, there are $3 \times 6-(16-1)=3$ absolute invariants under the action of general linear group $G L(3)$ in $\mathcal{P}^{3}$. Since each point in $\mathcal{P}^{3}$ has 3 degrees of freedom, the dimension of a set of 6 points is $3 \times 6=18$. The transformation group $G L(3)$ is represented by a $4 \times 4$ matrix up to a scaling factor, its dimension is $4 \times 4-1=15$.

These 3 invariants can be formed and interpreted differently. For instance, following invariant theory, invariants can be expressed by linear combinations of products of the determinants of 4 by 4 matrix whose columns are the homogeneous coordinates of the points. This is mainly a domain of symmetric functions of the coordinates of the points. The invariants formed this way can be symmetric, therefore independent of the order in which the points are taken. Sometimes, half symmetric functions are also used. Coble [24] was interested in studying the invariants of associated sets of points between spaces of different dimensions. Using the six half symmetrical Jourbet's functions, he investigated the complete system of the (relative) invariants of six points of different dimension. We will not go further into his work, as it is rather complicated and involves concepts beyond the scope of this paper.

Another way is to consider twisted cubics [25], since there is a unique twisted cubic that passes through the given 6 general points in $\mathcal{P}^{3}$. Any 4 points of a twisted cubic define a cross ratio, therefore a subordinate one-dimensional projective geometry is induced on any twisted cubic. So the 3 invariants of the set of 6 points can be taken as the 3 independent cross ratios of the sets of 4 points on the twisted cubic. Or more algebraically, we can consider the invariants of cubic forms. Cubic forms in $\mathcal{P}^{3}$ have 5 algebraically independent relative invariants of degree $8,16,24$, 32, 40 ( $c f$. [27]). Three absolute invariants can be derived from these 5 relative invariants. However the expressions for these invariants are very complicated polynomials. One of the simplest ways to consider these 3 invariants is by considering the 3 non-homogeneous projective coordinates of any sixth point with respect to a projective basis defined by any 5 of them. In the following, we will consider these invariants, since it seems to us that this is the simplest way to deal with them, and also because this methodology has been succeesfully used in [7], [1], [28], [8], [18], particularly by Faugeras in [1]. Obviously, these non homogeneous projective coordinates admit direct cross-ratio interpretation $[25]$.

\section{INVARIANT RELATIONSHIP OF SIX POINTS FROM ONE IMAGE}

\section{A. Canonical representation of 6 points in space}

Given any six points $\left\{P_{i}, i=1, \ldots, 6\right\}$ in $\mathcal{P}^{3}$, in view of the fundamental theorem for projective space, any five of them, no 3 of them collinear and no 4 of them coplanar, can be given preassigned coordinates, thus we can assign them the canonical projective coordinates as follows

$$
\begin{aligned}
& (1,0,0,0)^{T},(0,1,0,0)^{T},(0,0,1,0)^{T}, \\
& (0,0,0,1)^{T} \text { and }(1,1,1,1)^{T} .
\end{aligned}
$$

This uniquely determines a space collineation $A_{4 \times 4}$, $\operatorname{det}\left(A_{4 \times 4}\right) \neq 0$, which transforms the original 5 points into this canonical basis. And for the sixth point, it is transformed into its projective coordinates $(X, Y, Z, T)^{T}$ by $A_{4 \times 4}$. Therefore,

$X: Y: Z: T$ gives the 3 independent absolute invariants of 6 points.

\section{B. Canonical representation of 6 image points}

The projections of these six points onto an image $\left\{p_{i}, i=\right.$ $1, \ldots, 6\}$ are usually given in non-homogeneous coordinates

$$
\left(x_{i}, y_{i}\right)^{T}, i=1, \ldots, 6
$$

take any 4 of them, no 3 of them collinear, and assign them the canonical projective coordinates in $\mathcal{P}^{2}$

$$
(1,0,0)^{T},(0,1,0)^{T},(0,0,1)^{T} \text {, and }(1,1,1)^{T} \text {. }
$$

A plane collineation $A_{3 \times 3}, \operatorname{det}\left(A_{3 \times 3}\right) \neq 0$, can be uniquely determined. $A_{3 \times 3}$ transforms the fifth and sixth points into $\left(u_{5}, v_{5}, w_{5}\right)^{T}$ and $\left(u_{6}, v_{6}, w_{6}\right)^{T}$. Therefore, $u_{5}: v_{5}: w_{5}$ and $u_{6}: v_{6}: w_{6}$ give the 4 independent absolute invariants of 6 image points.

The algebraic determination of $A_{3 \times 3}$ can be found in Appendix.

\section{Projection between space and image plane}

If we assume a perspective projection as the camera model, then object space may be considered as embedded in $\mathcal{P}^{3}$ and image space embedded in $\mathcal{P}^{2}$. The camera performs the projection from $\mathcal{P}^{3}$ upon $\mathcal{P}^{2}$, and this projection can be represented by a $3 \times 4$ matrix $C_{3 \times 4}$ of rank 3 whose kernel is the projection center. The relation between the points $P_{i}$ in $\mathcal{P}^{3}$ and $p_{i}$ in $\mathcal{P}^{2}$ can be written as

$$
\lambda_{i} p_{i}=C_{3 \times 4} P_{i},
$$


where $p_{i}$ and $P_{i}$ are in homogeneous coordinates. i.e.

$$
\lambda_{i}\left(\begin{array}{c}
u_{i} \\
v_{i} \\
w_{i}
\end{array}\right)=\left(\begin{array}{cccc}
c_{11} & c_{12} & c_{13} & c_{14} \\
c_{21} & c_{22} & c_{23} & c_{24} \\
c_{31} & c_{32} & c_{33} & c_{34}
\end{array}\right)\left(\begin{array}{c}
X_{i} \\
Y_{i} \\
Z_{i} \\
T_{i}
\end{array}\right)
$$

This can be rewritten in ratio form hiding the scaling factor $\lambda_{i}$

$$
\begin{aligned}
u_{i}: v_{i}: w_{i}= & \left(c_{11} X_{i}+c_{12} Y_{i}+c_{13} Z_{i}+c_{14} T_{i}\right): \\
& \left(c_{21} X_{i}+c_{22} Y_{i}+c_{23} Z_{i}+c_{24} T_{i}\right): \\
& \left(c_{31} X_{i}+c_{32} Y_{i}+c_{33} Z_{i}+c_{34} T_{i}\right)
\end{aligned}
$$

For each point, as $u_{i}, v_{i}$ and $w_{i}$ can not all be zero, two independent equations can always be derived from (1). These equations express nothing else than the collinearity of the space points and their corresponding image points. This is a projective property which is preserved by any projective transformation.

The calibration process consists of the determination of all the parameters $c_{i j}$ of the projection matrix $C_{3 \times 4}$. As we are assuming that we are working with uncalibrated images, it is meant that $C_{3 \times 4}$ is totally unknown.

\section{Elimination of camera parameters $c_{i j}$}

For a set of 6 points in image and in space, when the correspondences $p_{i} \leftrightarrow P_{i}$, for $i=1, \ldots, 6$, are given as

$$
\begin{aligned}
& (1,0,0)^{T} \leftrightarrow(1,0,0,0)^{T}, \\
& (0,1,0)^{T} \leftrightarrow(0,1,0,0)^{T}, \\
& (0,0,1)^{T} \leftrightarrow(0,0,1,0)^{T}, \\
& (1,1,1)^{T} \leftrightarrow(0,0,0,1)^{T}, \\
& \left(u_{5}, v_{5}, w_{5}\right)^{T} \leftrightarrow(1,1,1,1)^{T}, \\
& \left(u_{6}, v_{6}, w_{6}\right)^{T} \leftrightarrow(X, Y, Z, T)^{T},
\end{aligned}
$$

this leads to $12=2 \times 6$ equations from (1). All entries $c_{i j}$ of $C_{3 \times 4}$ are unknowns, as we assumed that the camera was uncalibrated. Since $C_{3 \times 4}$ is defined up to a scaling factor, it counts for $3 \times 4-1=11$ unknowns. So there still remains one $(1=12-11)$ independent equation after eliminating all unknown camera parameters $c_{i j}$.

Substituting all canonical projective coordinates of the 6 points in image and in space into equations (1). Then eliminating $c_{i j}$, we obtain the following homogeneous equation between $X, Y, Z, T$ and $\left\{\left(u_{i}, v_{i}, w_{i}\right)\right.$, for $\left.i=5,6\right\}$,

$$
\begin{array}{llll}
w_{6}\left(u_{5}-v_{5}\right) X Y & +v_{6}\left(w_{5}-u_{5}\right) X Z & + & \\
u_{5}\left(v_{6}-w_{6}\right) X T & +u_{6}\left(v_{5}-w_{5}\right) Y Z & + & \\
v_{5}\left(w_{6}-u_{6}\right) Y T & +w_{5}\left(u_{6}-v_{6}\right) Z T & = & 0 .
\end{array}
$$

\section{E. Invariant interpretation of the equation}

The above equation (2) will be arranged and interpreted as an invariant relationship between the relative invariants of $\mathcal{P}^{3}$ and those of $\mathcal{P}^{2}$ as follows.

If $i_{j}$ and $I_{j}$ denote respectively

$$
\begin{aligned}
& i_{1}=w_{6}\left(u_{5}-v_{5}\right), \\
& i_{2}=v_{6}\left(w_{5}-u_{5}\right), \\
& i_{3}=u_{5}\left(v_{6}-w_{6}\right), \\
& i_{4}=u_{6}\left(v_{5}-w_{5}\right), \\
& i_{5}=v_{5}\left(w_{6}-u_{6}\right), \\
& i_{6}=w_{5}\left(u_{6}-v_{6}\right),
\end{aligned}
$$

and

$$
\begin{aligned}
& I_{1}=X Y, \quad I_{2}=X Z, \quad I_{3}=X T \\
& I_{4}=Y Z, \quad I_{5}=Y T, \quad I_{6}=Z T
\end{aligned}
$$

then $i_{j}$ and $I_{j}$ can be interpreted as respectively the relative invariants of 6 points in $\mathcal{P}^{2}$ (image) and those of 6 points in $\mathcal{P}^{3}$ (space).

$\left\{i_{j}, j=1, \ldots, 6\right\}$ are defined up to a common multiplier, so they are only relative invariants of the 6 points of the image. The 5 ratios $i_{1}: i_{2}: i_{3}: i_{4}: i_{5}: i_{6}$ are (absolute) projective invariants. Since (by the arguments of Section II) for the set of 6 points in $\mathcal{P}^{2}$, there are $4=2 \times 6-8$ independent projective invariants, therefore the relative invariants $\left\{i_{j}, j=1, \ldots, 6\right\}$ are not independent and are subject to one $(1=5-4)$ additional constraint which is

$$
i_{1}+i_{2}+i_{3}+i_{4}+i_{5}+i_{6}=0 \text {. }
$$

This can be checked from the above definition of $i_{j}$.

$\left\{I_{j}, j=1, \ldots, 6\right\}$ are relative invariants of $\mathcal{P}^{3}$, only the 5 ratios $I_{1}: I_{2}: I_{3}: I_{4}: I_{5}: I_{6}$ are projective invariants in space. Since there are only 3 independent absolute invariants, $I_{j}$ are subject to two additional constraints which are, by inspection of the definition of $I_{j}$,

$$
\frac{I_{1}}{I_{2}}=\frac{I_{5}}{I_{6}} \text { and } \frac{I_{2}}{I_{3}}=\frac{I_{4}}{I_{5}}
$$

An independent set of three absolute invariants in space could be taken to be

$$
\alpha \equiv \frac{I_{2}}{I_{6}}=\frac{X}{T}, \beta \equiv \frac{I_{4}}{I_{6}}=\frac{Y}{T} \text { and } \gamma \equiv \frac{I_{2}}{I_{3}}=\frac{Z}{T} .
$$

The set of invariants $\{\alpha, \beta, \gamma\}$ is equivalent to $X: Y: Z$ : $T$.

Then the invariant relation (2) is simply expressed as a bilinear homogeneous relation,

$$
i_{1} I_{1}+i_{2} I_{2}+i_{3} I_{3}+i_{4} I_{4}+i_{5} I_{5}+i_{6} I_{6}=0 .
$$

This relationship is of course independent of any camera parameters, therefore it can be used for any uncalibrated images. This clearly shows that the $3 \mathrm{D}$ invariants can not be computed from one single image, since we have only one invariant relationship for three independent invariants. This invariant relationship can be used in two different ways:

- to verify whether a given set of points is present in a model base. That means $I_{j}$ can be computed from the model base and $i_{j}$ from the image, then the invariant relation can be used to check for the presence of the model in the image.

- to compute 3D invariants from more than one image, this leads to the following section. 
IV. Computation of the invariants of SiX Points FROM THREE IMAGES

In this section, we will focus on how to find the absolute invariants of 6 points in space, i.e. $\{\alpha, \beta, \gamma\}$ or equivalently $X: Y: Z: T$ from more than one image. To do this, it is assumed that the 6 point correspondences through images have already been established.

So from a set of six point in one image, the homogeneous invariant relation (3) can be written in $X, Y, Z, T$ as

$$
i_{1} X Y+i_{2} X Z+i_{3} X T+i_{4} Y Z+i_{5} Y T+i_{6} Z T=0,
$$

with coefficients $i_{j}$ computed from image points. As there are only 3 independent invariants for a set of 6 points, we might hope to solve for these 3 invariants if 3 images of the set of 6 points are available. The three homogeneous quadratic equations in $X, Y, Z, T$ from three images can be written as follows.

$$
\begin{aligned}
\mathcal{F}_{1} \equiv & i_{1}^{(1)} X Y+i_{2}^{(1)} X Z+i_{3}^{(1)} X T \\
& +i_{4}^{(1)} Y Z+i_{5}^{(1)} Y T+i_{6}^{(1)} Z T=0 \\
\mathcal{F}_{2} \equiv & i_{1}^{(2)} X Y+i_{2}^{(2)} X Z+i_{3}^{(2)} X T \\
& +i_{4}^{(2)} Y Z+i_{5}^{(2)} Y T+i_{6}^{(2)} Z T=0 \\
\mathcal{F}_{3} \equiv & i_{1}^{(3)} X Y+i_{2}^{(3)} X Z+i_{3}^{(3)} X T \\
& +i_{4}^{(3)} Y Z+i_{5}^{(3)} Y T+i_{6}^{(3)} Z T=0
\end{aligned}
$$

here the superscripts $(j), j=1,2,3$, distinguish the invariant quantities of different images.

\section{A. Maximum number of possible solutions}

Each equation represents a quadratic surface which has rank 3. These quadratic forms have no $X^{2}, Y^{2}, Z^{2}, T^{2}$ terms. That means that the quadratic surface goes through the vertices of the tetrahedron of reference whose coordinates are $(0,0,0,1)^{T},(0,0,1,0)^{T},(0,1,0,0)^{T},(1,0,0,0)^{T}$. This is easily verified by substituting these points in the equations. In addition, as the coefficients of the quadratic form $i^{(j)}$ are subject to the following relations,

$$
i_{1}^{(j)}+i_{2}^{(j)}+i_{3}^{(j)}+i_{4}^{(j)}+i_{5}^{(j)}+i_{6}^{(j)}=0, \text { for } j=1,2,3,
$$

so all the equations (4), (5) and (6) necessarily pass through the unit point $(1,1,1,1)^{T}$.

According to Bezout's Theorem, three quadratic surfaces must meet in $8=2 \times 2 \times 2$ points. Since they already pass through the five known points, so only $3=8-5$ common points remain. Therefore,

the maximum number of solutions for $X: Y: Z: T$ is three.

\section{B. Find $X / T$ by solving a cubic equation}

Now, let us try to explicit these 3 solutions. For instance, if we want to solve for $X / T$, the two resultants $\mathcal{G}_{1}$ and $\mathcal{G}_{2}$ obtained by eliminating $Z$ between $\mathcal{F}_{1}$ and $\mathcal{F}_{3}$ and between $\mathcal{F}_{2}$ and $\mathcal{F}_{3}$ are homogeneous polynomials in $X, Y$ and $T$ of degree 3 :

$$
\begin{aligned}
\mathcal{G}_{1} \equiv & e_{1}^{(1)} X^{2} Y+e_{2}^{(1)} X Y^{2}+e_{3}^{(1)} X Y T+e_{4}^{(1)} X^{2} T \\
& +e_{5}^{(1)} X T^{2}+e_{6}^{(1)} Y^{2} T+e_{7}^{(1)} Y T^{2}=0, \\
\mathcal{G}_{2} \equiv & e_{1}^{(2)} X^{2} Y+e_{2}^{(2)} X Y^{2}+e_{3}^{(2)} X Y T+e_{4}^{(2)} X^{2} T \\
& +e_{5}^{(2)} X T^{2}+e_{6}^{(2)} Y^{2} T+e_{7}^{(2)} Y T^{2}=0,
\end{aligned}
$$

whose coefficients are still subject to

$e_{1}^{(j)}+e_{2}^{(j)}+e_{3}^{(j)}+e_{4}^{(j)}+e_{5}^{(j)}+e_{6}^{(j)}+e_{7}^{(j)}=0$ for $j=1,2$,

where

$$
\begin{aligned}
& e_{1}^{(j)}=i_{4}^{(j+1)} i_{5}^{(j)}-i_{4}^{(j)} i_{5}^{(j+1)}, \\
& e_{2}^{(j)}=i_{4}^{(j+1)} i_{6}^{(j)}-i_{4}^{(j)} i_{6}^{(j+1)}, \\
& e_{4}^{(j)}=i_{1}^{(j+1)} i_{5}^{(j)}-i_{1}^{(j)} i_{5}^{(j+1)}, \\
& e_{5}^{(j)}=i_{1}^{(j+1)} i_{3}^{(j)}-i_{1}^{(j)} i_{3}^{(j+1)}, \\
& e_{6}^{(j)}=i_{2}^{(j+1)} i_{6}^{(j)}-i_{2}^{(j)} i_{6}^{(j+1)}, \\
& e_{7}^{(j)}=i_{2}^{(j+1)} i_{3}^{(j)}-i_{2}^{(j)} i_{3}^{(j+1)} .
\end{aligned}
$$

This is due to the fact that the point $(1,1,1)^{T}$ is still on the two cubic curves. The resultant obtained by eliminating $Y$ between $\mathcal{G}_{1}$ and $\mathcal{G}_{2}$ is a homogeneous polynomial in $X, T$ of degree 8 , which can be factorized as,

$$
\begin{array}{r}
X T(X-T)\left(b_{1} X^{2}+b_{2} X T+b_{3} T^{2}\right)\left(a_{1} X^{3}\right. \\
\left.+a_{2} X^{2} T+a_{3} X T^{2}+a_{4} T^{3}\right) .
\end{array}
$$

It is evident that the linear factors lead to trivial solutions. The solution $X=0$ corresponds to the common points $(0,0,0,1)^{T},(0,0,1,0)^{T}$ and $(0,1,0,0)^{T}$. The solution $T=0$ corresponds to the common points $(1,0,0,0)^{T}$, $(0,1,0,0)^{T}$ and $(0,0,1,0)^{T}$. The solution $X=T$ corresponds to the common point $(1,1,1,1)^{T}$.

Now, let us have a close-look at the coefficients of the quadratic factor $b_{1} X^{2}+b_{2} X T+b_{3} T^{2}$,

$$
\begin{aligned}
& b_{1}=i_{4}^{(2)} i_{5}^{(2)} \\
& b_{2}=i_{3}^{(2)} i_{4}^{(2)}+i_{2}^{(2)} i_{5}^{(2)}-i_{1}^{(2)} i_{6}^{(2)}, \\
& b_{3}=i_{2}^{(2)} i_{3}^{(2)}
\end{aligned}
$$

which depend only on the invariant quantities of the points of the second image, so the zeros of the quadratic factor are the parasite solutions introduced by elimination using resultants, they are not the zeros of $\left\{\mathcal{F}_{1}, \mathcal{F}_{2}, \mathcal{F}_{3}\right\}$. Thus, the only nontrivial solutions for $X / T$ are those of the cubic equation,

$$
\mathcal{C} \equiv a_{1} X^{3}+a_{2} X^{2} T+a_{3} X T^{2}+a_{4} T^{3}=0 .
$$

The implicit expressions for $a_{i}$ (quite long) can be easily obtained with Maple. The cubic equation may be solved algebraically by Cardano's formula, either for $X / T$ or $T / X$. The choice between solving $X / T$ or $T / X$ depends on the estimation of the moduli of the roots. According to Cauchy's Theorem, the moduli of the roots of cubic equations are bounded by the following,

$$
\left(1+\frac{\max \left\{\left|a_{2}\right|,\left|a_{4}\right|\right\}}{\left|a_{1}\right|}\right)^{-1}<\left|\frac{X}{T}\right|<1+\frac{\max \left\{\left|a_{1}\right|,\left|a_{3}\right|\right\}}{\left|a_{4}\right|} .
$$

If the modulus is large enough, that means $\mathrm{T}$ is near to 0 , in this case it would be better to solve for its reciprocal $T / X$ instead of $X / T$. 
C. Find $Y: Z: T$ linearly for a given $X / T$

$Y: Z: T$ should be solved for each given $X: T$. As the equations are symmetric in $X, Y, Z$ and $T$, we could obtain other similar cubic equations for the other two independent absolute invariants, for instance, $Y / T$ and $Z / T$ or any other ratios if necessary. However, doing this would lead to much more than 3 solution sets which is not desirable. We expect to obtain unique $Y: Z: T$ for each given $X: T$.

By eliminating $Y^{2}$ between $\mathcal{G}_{1}$ and $\mathcal{G}_{2}$, we obtain the following homogeneous polynomial

$$
\begin{aligned}
\mathcal{H} \equiv & \left(c_{1} X^{3}+c_{2} X^{2} T+c_{3} X T^{2}+c_{4} T^{3}\right) Y \\
& +X\left(d_{1} X^{2}+d_{2} X T+d_{3} T^{2}\right) T
\end{aligned}
$$

where

$$
\begin{aligned}
& c_{1}=e_{2}^{(2)} e_{1}^{(1)}-e_{1}^{(2)} e_{2}^{(1)}, \\
& c_{2}=e_{6}^{(2)} e_{1}^{(1)}-e_{3}^{(2)} e_{2}^{(1)}+e_{2}^{(2)} e_{3}^{(1)}-e_{1}^{(2)} e_{6}^{(1)}, \\
& c_{3}=e_{6}^{(2)} e_{3}^{(1)}-e_{7}^{(2)} e_{2}^{(1)}-e_{3}^{(2)} e_{6}^{(1)}+e_{2}^{(2)} e_{7}^{(1)}, \\
& c_{4}=e_{6}^{(2)} e_{7}^{(1)}-e_{7}^{(2)} e_{6}^{(1)}
\end{aligned}
$$

and

$$
\begin{aligned}
& d_{1}=e_{2}^{(2)} e_{4}^{(1)}-e_{4}^{(2)} e_{2}^{(1)} \\
& d_{2}=e_{2}^{(2)} e_{5}^{(1)}+e_{6}^{(2)} e_{4}^{(1)}-e_{5}^{(2)} e_{2}^{(1)}-e_{4}^{(2)} e_{6}^{(1)} \\
& d_{3}=-e_{5}^{(2)} e_{6}^{(1)}+e_{6}^{(2)} e_{5}^{(1)} .
\end{aligned}
$$

Any zero of $\mathcal{H}$ is also a zero of $\mathcal{G}_{1}$ and $\mathcal{G}_{2}$, as $\mathcal{H}$ is a linear combination of $\mathcal{G}_{1}$ and $\mathcal{G}_{2}$ with polynomial coefficients, and $\mathcal{H}$ belongs to the ideal generated by $\mathcal{G}_{1}$ and $\mathcal{G}_{2}$.

$\mathcal{H}$ can be considered as a linear homogeneous polynomial in $Y$ and $T$ for a given $X / T$. Therefore a unique $Y: T$ is guaranteed from this equation for each given $\alpha=X: T$,

$$
Y: T=-\alpha\left(d_{1} \alpha^{2}+d_{2} \alpha+d_{3}\right):\left(c_{1} \alpha^{3}+c_{2} \alpha^{2}+c_{3} \alpha+c_{4}\right) .
$$

For a given $X: Y: T$, say $\alpha=X / T$ and $\beta=Y / T, Z: T$ is uniquely determined by one of the equations (4), (5) or (6), for instance taking (4), we have

$$
Z: T=-\left(i_{1}^{(1)} \alpha \beta+i_{3}^{(1)} \alpha+i_{5}^{(1)} \beta\right):\left(i_{2}^{(1)} \alpha+i_{4}^{(1)} \beta+i_{6}^{(1)}\right) .
$$

In conclusion, only at most three solutions exist for $X: Y$ : $Z: T$, hence we establish the following.

Given a set of 6 point correspondences in 3 images taken by uncalibrated cameras, there are at most 3 solutions for the three invariants associated to the set of 6 points in space, such solutions can be found in closed form.

\section{Summary of computation}

The computation of the invariants of six points can be summarized in the following simple algorithm.

1. Compute the plane collineation $A_{3 \times 3}$ ( $c f$. Appendix) such that 4 of the 6 points of each image go to the canonical projective basis. Apply this collineation to the remaining two points to obtain

$$
\left\{u_{i}^{(j)}, v_{i}^{(j)}, w_{i}^{(j)}, \text { for } i=5,6 \text { and } j=1,2,3\right\}
$$

2. Compute the relative invariants of each image

$$
\left\{i_{k}^{(j)}, \text { for } j=1,2,3 \text { and } k=1, \ldots 6\right\}
$$

from the above transformed image points;

3. Solve the cubic equation (9) $\mathcal{C}=0$ for $\alpha=X / T$;

4. For each $\alpha$, solve the linear equation (10) $\mathcal{H}=0$ for $\beta=Y / T$

5. For each $\alpha$ and $\beta$, solve the linear equation (4) $\mathcal{F}_{1}=0$ for $\gamma=Z / T$.

\section{E. Remark}

We can note that instead of solving $\left\{\mathcal{F}_{1}, \mathcal{F}_{2}, \mathcal{F}_{3}\right\}$ for $X$ : $Y: Z: T$, we can equivalently solve

$$
\left\{\begin{array}{l}
i_{1}^{(1)} I_{1}+i_{2}^{(1)} I_{2}+i_{3}^{(1)} I_{3}+i_{4}^{(1)} I_{4}+i_{5}^{(1)} I_{5}+i_{6}^{(1)} I_{6}=0 \\
i_{1}^{(2)} I_{1}+i_{2}^{(2)} I_{2}+i_{3}^{(2)} I_{3}+i_{4}^{(2)} I_{4}+i_{5}^{(2)} I_{5}+i_{6}^{(2)} I_{6}=0 \\
i_{1}^{(3)} I_{1}+i_{2}^{(3)} I_{2}+i_{3}^{(3)} I_{3}+i_{4}^{(3)} I_{4}+i_{5}^{(3)} I_{5}+i_{6}^{(3)} I_{6}=0 \\
I_{1} I_{6}-I_{2} I_{5}=0 \\
I_{1} I_{6}-I_{3} I_{4}=0
\end{array}\right.
$$

for the ratios $I_{1}: I_{2}: I_{3}: I_{4}: I_{5}: I_{6}$.

They meet in $4=1 \times 1 \times 1 \times 2 \times 2$ intersection points in $\mathcal{P}^{5}$. As the sum of the coefficients is zero, they meet in the common unit point $(1,1,1,1,1,1)^{T}$, so only 3 solutions remain. The same results, cubic equation, can be obtained by a little more symbolic computation.

We also note that when 5 images are available, a linear solution for the ratios $I_{1}: I_{2}: I_{3}: I_{4}: I_{5}: I_{6}$ is possible while ignoring the quadratic constraints $I_{1} I_{6}=I_{2} I_{5}=I_{3} I_{4}$.

\section{Applications of the invariants}

In this section, we are exploring some interesting applications that may be provided by the computed invariants of six points. The most important is undoubtly the complete determination of the projection matrices of the three cameras. This gives the most complete projectively invariant description of the three cameras. It follows that projective reconstruction as has been achieved by Faugeras [1] and Hartley et al. [2] and the determination of epipolar transformation by Sturm's method [3] are extended to the case of three uncalibrated cameras with fewer points.

\section{A. Complete determination of projection matrices $C_{3 \times 4}^{j}$}

In the case of two uncalibrated cameras, Faugeras [1] used epipolar geometry to determine the projection matrix up to projective transformations. Here, in the case of three uncalibrated images, as the projective coordinates of the sixth point is algebraically determined. It means that 6 points both in $\mathcal{P}^{2}$ and $\mathcal{P}^{3}$ are known up to respectively a plane and a space collineation. It turns out that the projection matrix $C_{3 \times 4}^{(j)}$ for each camera can be completely determined.

For each camera, the correspondences of 6 points between $\mathcal{P}^{2}$ and $\mathcal{P}^{3}$ result in $2 \times 6=12$ linear homogeneous equations in the $3 \times 4=12$ entries $c_{i j}$ that count only for 11 unknowns due to a common scaling factor. One of 
the 12 equations has been used to derive the invariant relationship (3) ( $c f$. Section III-D), which was then used to compute the invariants. Therefore, there remain only $12-1=11$ independent linear equations which matches the 11 unknowns of the projection matrix. After some simple algebra, the projection matrix for the $j$-th camera can be written as follows,

$$
C_{3 \times 4}^{(j)}=\left(\begin{array}{cccc}
c_{11}^{(j)} & 0 & 0 & 1 \\
0 & c_{22}^{(j)} & 0 & 1 \\
0 & 0 & c_{33}^{(j)} & 1
\end{array}\right)
$$

where

$$
\begin{aligned}
c_{11}^{(j)} & =u_{5}^{(j)} k^{(j)}-1, \\
c_{22}^{(j)} & =v_{5}^{(j)} k^{(j)}-1, \\
c_{33}^{(j)} & =w_{5}^{(j)} k^{(j)}-1
\end{aligned}
$$

and $k^{(j)}$ can be taken as either

$$
\frac{\left(u_{6}^{(j)}-w_{6}^{(j)}\right)+w_{6}^{(j)} \alpha-u_{6}^{(j)} \gamma}{u_{5}^{(j)} w_{6}^{(j)} \alpha-u_{6}^{(j)} w_{5}^{(j)} \gamma}
$$

or

$$
\frac{\left(u_{6}^{(j)}-v_{6}^{(j)}\right)+v_{6}^{(j)} \alpha-u_{6}^{(j)} \beta}{u_{5}^{(j)} v_{6}^{(j)} \alpha-u_{6}^{(j)} v_{5}^{(j)} \beta},
$$

which are equivalent due to the invariant relationship (3).

\section{B. Projective reconstruction}

The complete determination of projection matrices implies that given any point correspondence in images, the corresponding point in space can be reconstructed in $\mathcal{P}^{3}$ up to a collineation. In fact, projective reconstruction is equivalent to the computation of the projective invariants of the sets of points.

Given the point correspondences $\left(u_{i}^{(j)}, v_{i}^{(j)}, w_{i}^{(j)}\right)^{T}$ and the projection matrices $C_{3 \times 4}^{(j)}$, for $j=1,2,3$, we can write two linear homogeneous equations for the unknows $X_{i}, Y_{i}, Z_{i}, T_{i}$ of each point for each image as follows,

$$
\left\{\begin{array}{l}
w_{i}^{(j)} c_{11}^{(j)} X_{i}-u_{i}^{(j)} c_{33}^{(j)} Z_{i}+\left(w_{i}^{(j)}-u_{i}^{(j)}\right) T_{i}=0 \\
w_{i}^{(j)} c_{22}^{(j)} Y_{i}-v_{i}^{(j)} c_{33}^{(j)} Z_{i}+\left(w_{i}^{(j)}-v_{i}^{(j)}\right) T_{i}=0 .
\end{array}\right.
$$

With three images $j=1,2,3$, a linear system of 6 homogeneous equations is obtained. An algebraic solution can be found by solving any three of them. A numerical solution can also be found by solving this over-determined linear system. Therefore we establish the following result which can be considered as an extension to the recent work of Faugeras [1] and Hartley et al. [2] for reconstruction from two uncalibrated images.

Given a set of $n \geq 6$ point correspondences in 3 images taken by uncalibrated cameras, the set of points can be reconstructed in $\mathcal{P}^{3}$ up to a collineation.

The reconstruction may have as many as three different solutions from the minimal data of six points.

Notice that this result reduces the number of points needed to have a projective reconstruction to the strict minimum of six.

\section{Determination of the epipolar geometry}

Another consequence of the complete determination of the projection matrices is that the epipolar geometry of any pair of the three cameras can be computed with only 6 point correspondences.

Given a set of 6 point correspondences in 3 images taken by uncalibrated cameras, the epipolar geometry of any pair of cameras can be determined, up to at most 3 solutions.

This can be compared with Sturm's method [29], [3], reintroduced into computer vision in [30], [21], [19], which is of great importance for two uncalibrated cameras. Based on projective geometry, Sturm's method yields at most three solutions in general for the epipolar geometry, which are three of the nine points of intersection of two cubic plane curves.

Note that although the two approaches developed respectively for the two-camera and the three-camera cases lead to equivalent results, the parametrizations used in two cases are different. Sturm directly used the epipolar transformation as the parameters for the two uncalibrated cameras, while we used the projective structure of the point set in space as parameters for the three uncalibrated cameras. The underlying equations of the parametrization for the three-camera case are simpler, hence a better numerical stability might be expected. This is to be confirmed by the experimental results presented in Section VI.

For extracting the epipolar geometry from any given pair of projection matrices, one can refer, for instance, to [1] for more details. Here we are content with the following results.

- The epipole in the $j$-th image with respect to the $i$-th camera $e_{j i}$ is given by

$$
\begin{aligned}
e_{j i} & =C_{3 \times 4}^{(j)} \operatorname{ker}\left(C_{3 \times 4}^{(i)}\right) \\
& =\left(1-h_{1}, 1-h_{2}, 1-h_{3}\right)^{T}
\end{aligned}
$$

where

$$
h_{1}=\frac{c_{11}^{(j)}}{c_{11}^{(i)}}, \quad h_{2}=\frac{c_{22}^{(j)}}{c_{22}^{(i)}}, \quad h_{3}=\frac{c_{33}^{(j)}}{c_{33}^{(i)}} .
$$

- The epipolar geometry between $i$-th and $j$-th cameras, represented by the fundamental matrix $F_{i j}$ [19] (the generalization of the essential matrix [23]) is given by

$$
\begin{aligned}
F_{i j} & =\left[e_{j i}\right] C_{3 \times 3}^{(j)}\left(C_{3 \times 3}^{(i)}\right)^{-1} \\
& =\left(\begin{array}{ccc}
0 & -h_{2}\left(h_{3}-1\right) & h_{3}\left(h_{2}-1\right) \\
h_{1}\left(h_{3}-1\right) & 0 & -h_{3}\left(h_{1}-1\right) \\
-h_{1}\left(h_{2}-1\right) & h_{2}\left(h_{1}-1\right) & 0
\end{array}\right)
\end{aligned}
$$

where $\left[e_{j i}\right]$ is the anti-symmetric matrix associated to $e_{j i}$, and $C_{3 \times 3}$ is the left $3 \times 3$ sub-matrix of the $3 \times 4$ matrix $C_{3 \times 4}$.

\section{Camera self-calibration}

The above results can also be applied to the camera selfcalibration proposed by Maybank and Faugeras [21]. According to [21], [19], [20], from point correspondences of 
three uncalibrated images and the associated epipolar geometries, one can calibrate and therefore obtain a Euclidean reconstruction (only up to a global scaling factor), if we suppose that all three images are taken by the same uncalibrated camera.

Using Sturm's method or other equivalent algebraic methods for estimating the epipolar geometries for each pair of three cameras, one needs a minimum number of seven points to do that. While using the method presented above which considers the three cameras as a whole, the epipolar geometries of any pair of three cameras can be computed with only six points. Therefore, we can make the following statement.

A camera can be self-calibrated with a minimum of 6 point correspondences in 3 images.

\section{EXPERIMENTAL RESUlts}

The theoretical results presented above for the computation of the invariants of 6 points have been implemented. The accuracy and stability of the invariants with respect to various factors such as pixel errors, selection of the triplet of images and positions of the camera are studied both for simulated and real images. The computation should be accurate and stable in order for the invariants to be useful for vision applications. The three invariants used for experiments are $\alpha=X: T, \beta=Y: T$ and $\gamma=Z: T$. The application for projective reconstruction is also presented.

\section{A. The experiment with simulated images}

\section{A.1 Simulation set-up}

We will first simulate a number of images in which a set of 6 points in space is projected. The simulation is set up as follows.

- First, a real camera is calibrated (cf. [31], [6], [32]). This is done by placing a known object of about $50 \mathrm{~cm}^{3}$ in front of the camera. The camera is then moved through 9 different viewing positions, spaced at 10 degree intervals around the calibration object. The intervals between the first 3 views are slightly bigger than the second 3 views, and the second 3 views bigger than the third 3 ones. This creates 9 calibration matrices which are subsequently used in the simulation.

- With these 9 calibration matrices as our perfect cameras, we project several sets of 6 known points into these 9 synthetic images.

- Finally the projected positions of the points in the images are perturbed by varying levels of noise of uniform distribution.

- As the positions of the 6 simulated points in space are known in advance, their invariants are computed by the method given in Section II. These invariants are compared with those calculated from the simulated image data.

In this way, the realism of simulation is preserved, and the image noise can be quantitatively controlled as well in order to observe its influence.

The results that will be presented in the following tables are obtained from the set of 6 points whose (Euclidean) coordinates are $(2.0,0.0,12.0)^{T}(0.0,6.0,0.0)^{T}(12.0,0.0,14.0)^{T}$
$(0.0,6.0,6.0)^{T}(-1.5,19.5,0.0)^{T}$ and $(0.0,12.0,12.0)^{T}$ (the unit is in $\mathrm{cm}$ ). The other sets of 6 points are also simulated with the same calibration matrices and they have similar numerical behavior and will not be presented in the tables.

\section{A.2 Stability w.r.t. the selection of the triplets of images}

Table I shows the effect of choosing different triplets of synthetic images on the stability of the 3 invariants. All images points are perturbed by a uniform noise of \pm 1.5 pixels. In the case where the solution is unique, the computed value is followed by $\mathrm{a} *$ in the tables. When multiple solutions occur, the one that is the closest to the ground truth value is selected.

Another experimentation was carried out to test the stability with respect to the change of one of the 3 images. Four images 4, 5, 6 and 7 are taken, then all triplets are selected among the four. Here pixel error is set to \pm 1.5 . In the case of multiple solutions, all 3 possible solutions are given for each invariant, the solution that corresponds to the real solution is marked in bold font in Table II. From this table, we note that sometimes only one real solution is possible, in this case, it is the unique correct solution. In the case of existence of multiple solutions, the real solution is always present independent of the selection of the triplet and remains numerically stable.

TABLE II

TABLE OF INVARIANTS COMPUTED FROM DIFFERENT TRIPLETS OF SYNTHETIC IMAges. WITH \pm 1.5 PIXEL NOISE FOR IMAGE POINTS, ALL 3 SOLUTIONS OF $\{\alpha, \beta, \gamma\}$, IF THERE ARE, ARE SHOWN IN ORDER TO ILLUSTRATE THEIR STABILITY.

\begin{tabular}{|c|c|c|c|}
\hline \hline Image triplet & $\alpha=0.526421$ & $\beta=1.880620$ & $\gamma=0.745762$ \\
\hline & -3.779942 & 0.424841 & -1.588062 \\
$4,6,7$ & $\mathbf{0 . 5 1 5 3 7 2}$ & $\mathbf{1 . 8 9 9 4 9 6}$ & $\mathbf{0 . 7 4 4 1 7 6}$ \\
& 15.791148 & -0.023215 & -8.050262 \\
\hline $4,5,6$ & $\mathbf{0 . 5 1 6 4 7 6}$ & $\mathbf{1 . 8 9 9 0 5 6}$ & $\mathbf{0 . 7 4 7 5 5 5}$ \\
\hline & -2.006199 & 0.438052 & -1.051868 \\
& $\mathbf{0 . 5 1 5 0 8 0}$ & $\mathbf{1 . 8 9 9 5 9 3}$ & $\mathbf{0 . 7 4 2 2 0 3}$ \\
& 12.874219 & -0.414817 & -13.899986 \\
\hline $4,5,7$ & $\mathbf{0 . 5 1 6 0 1 4}$ & $\mathbf{1 . 8 9 9 5 5 1}$ & $\mathbf{0 . 7 4 6 5 6 4}$ \\
\hline
\end{tabular}

A.3 Stability w.r.t. the pixel errors

For the given triplets of images $\{1,2,3\},\{4,5,6\}$ and $\{7,8,9\}$, different pixel noise (uniformly distributed) is added to illustrate the influence of the pixel errors.

Tables III, IV and V show that with noise levels up to \pm 5.5 pixels, the computed invariants remain numerically stable, so that degradation with the increasing pixel noise is graceful.

Tables III, IV and V also show that the same invariant computed with completely different triplets of images remains stable.

From these empirical results, we note that the uniqueness of solutions depends partly on the positions of the cameras, for the first and the third triplet, the solutions are always multiple. For the second one, they are always unique up to \pm 3.5 pixel error. 


\section{TABLE I}

TABle OF INVARIANTS COMPUTED FROM DIFFERENT TRIPLETS OF SYNTHETIC IMAGeS. The POINTS OF IMAGeS ARE NOISED BY \pm 1.5 PIXEL

ERROR, THE INVARIANTS $\alpha, \beta$ AND $\gamma$ ARE COMPUTED FOR EACH DIFFERENT IMAGE TRIPLET. $d_{i}$ ARE THE DIFFERENCES BETWEEN EACH COMPUTED INVARIANT AND ITS MEAN VALUE. $\sigma$ IS THE STANDARD DEVIATION AND $\sigma / \bar{m}$ IS THE RATIO OF THE STANDARD DEVIATION TO THE MEAN VALUe.

\begin{tabular}{|c||c|c||c|c||c|c||}
\hline \hline Image triplet & $\alpha=0.526421$ & $d_{1}$ & $\beta=1.880620$ & $d_{2}$ & $\gamma=0.745762$ & $d_{3}$ \\
\hline $1,2,3$ & 0.528183 & 0.0083 & 1.877117 & -0.018 & 0.760771 & 0.0050 \\
\hline $4,5,6$ & $0.516476^{*}$ & -0.0034 & $1.899056^{*}$ & 0.0035 & $0.747555^{*}$ & -0.0082 \\
\hline $1,3,5$ & 0.524387 & 0.0045 & 1.889926 & -0.0056 & $0.760802^{*}$ & 0.0051 \\
\hline $5,7,9$ & $0.514523^{*}$ & -0.0053 & $1.899576^{*}$ & 0.0041 & $0.739840^{*}$ & -0.016 \\
\hline $1,4,8$ & 0.520458 & -0.00053 & 1.900477 & 0.0050 & 0.763048 & 0.0073 \\
\hline $2,5,8$ & 0.516762 & -0.0032 & 1.900178 & 0.0047 & 0.751696 & -0.0040 \\
\hline $1,5,9$ & 0.518681 & -0.0012 & 1.902235 & 0.0067 & 0.766406 & 0.011 \\
\hline mean $\bar{m}$ & 0.519924 & - & 1.895509 & - & 0.755731 & - \\
\hline$\sigma$ & 0.0048 & - & 0.0090 & - & 0.0096 & - \\
\hline$\sigma / m$ & 0.0092 & - & 0.0047 & - & 0.013 & - \\
\hline Ground truth & 0.526421 & - & 1.880620 & - & 0.745762 & - \\
\hline \hline
\end{tabular}

TABLE III

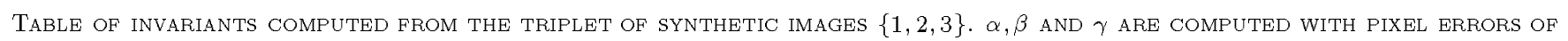
DIFFERENT LEVELS. $d_{i}$ IS THE DIFFERENCE BETWEEN THE COMPUTED VALUE AND ITS MEAN VALUE.

\begin{tabular}{|c||c|c||c|c||c|c||}
\hline \hline Noise & $\alpha=0.526421$ & $d_{1}$ & $\beta=1.880620$ & $d_{2}$ & $\gamma=0.745762$ & $d_{3}$ \\
\hline \pm 0.5 & 0.527054 & 0.00063 & 1.879408 & -0.0012 & 0.751121 & 0.0054 \\
\hline \pm 1.5 & 0.528183 & 0.0018 & 1.877117 & -0.0035 & 0.760771 & 0.015 \\
\hline \pm 2.5 & 0.529135 & 0.0027 & 1.875013 & -0.0056 & 0.769214 & 0.023 \\
\hline \pm 3.5 & 0.529938 & 0.0035 & 1.873026 & -0.0076 & 0.776658 & 0.031 \\
\hline \pm 4.5 & 0.530609 & 0.0042 & 1.871119 & -0.0095 & 0.783268 & 0.038 \\
\hline \pm 5.5 & 0.531163 & 0.0047 & 1.869260 & -0.011 & 0.789171 & 0.043 \\
\hline \hline
\end{tabular}

TABLE IV

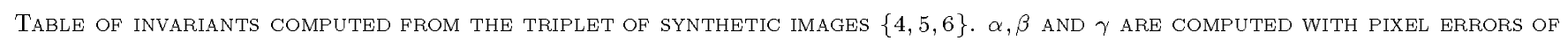
DIFFERENT LEVELS. $d_{i}$ IS THE DIFFERENCE BETWEEN THE COMPUTED VALUE AND ITS MEAN VALUE.

\begin{tabular}{|c||c|c||c|c||c|c||}
\hline \hline Noise & $\alpha=0.526421$ & $d_{1}$ & $\beta=1.880620$ & $d_{2}$ & $\gamma=0.745762$ & $d_{3}$ \\
\hline \pm 0.5 & $0.523211^{*}$ & -0.0032 & $1.886847^{*}$ & 0.0062 & $0.746559^{*}$ & 0.00080 \\
\hline \pm 1.5 & $0.516476^{*}$ & -0.0099 & $1.899056^{*}$ & 0.018 & $0.747555^{*}$ & 0.0018 \\
\hline \pm 2.5 & $0.509556^{*}$ & -0.017 & $1.911075^{*}$ & 0.030 & $0.748065^{*}$ & 0.0023 \\
\hline \pm 3.5 & $0.502432^{*}$ & -0.023 & $1.922747^{*}$ & 0.042 & $0.748102^{*}$ & 0.0023 \\
\hline \pm 4.5 & 0.495112 & -0.031 & 1.934272 & 0.054 & 0.747731 & 0.0020 \\
\hline \pm 5.5 & 0.487509 & -0.039 & 1.945445 & 0.065 & 0.746875 & 0.0011 \\
\hline \hline
\end{tabular}

TABLE V

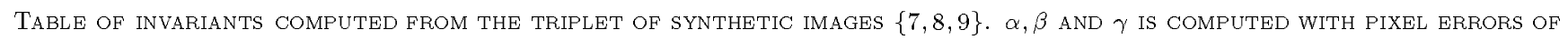

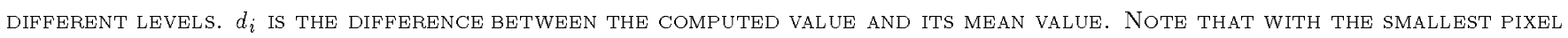
ERROR \pm 0.5 , THE RESULTS ARE NOT THE BEST ONES.

\begin{tabular}{|c||c|c||c|c||c|c||}
\hline \hline Noise & $\alpha=0.526421$ & $d_{1}$ & $\beta=1.880620$ & $d_{2}$ & $\gamma=0.745762$ & $d_{3}$ \\
\hline \pm 0.5 & 0.532012 & 0.0056 & 1.921869 & 0.041 & 0.710370 & -0.035 \\
\hline \pm 1.5 & 0.512018 & -0.014 & 1.858706 & -0.022 & 0.743526 & -0.0022 \\
\hline \pm 2.5 & 0.523644 & -0.0028 & 1.845212 & -0.035 & 0.727474 & -0.018 \\
\hline \pm 3.5 & 0.496563 & -0.030 & 1.930408 & 0.050 & 0.718309 & -0.027 \\
\hline \pm 4.5 & 0.502496 & -0.024 & 1.966565 & 0.086 & 0.721738 & -0.024 \\
\hline \pm 5.5 & 0.501878 & -0.025 & 1.964701 & 0.084 & 0.717939 & -0.028 \\
\hline \hline
\end{tabular}

\section{A.4 Stability w.r.t. the distance between cameras}

In Table I, the different triplets are ordered in increasing camera distance order, globally, the accuracy of the computed invariants is slightly improved by increasing camera distance.

Although the camera distance decreases slightly from $\{1,2,3\}$ to $\{4,5,6\}$ and $\{7,8,9\}$, comparing Tables III, IV and $\mathrm{V}$, the difference of the accuracy of the computed in- variants is not significant, this is due to the fact that the camera distance for those triplets is already favorable.

In the next section, camera distance influence will be discussed with real image sequences.

\section{$B$. The experiment with real images}

We first experimented over a sequence of 24 real images. The object spans about $30 \mathrm{~cm}$ in space and is placed in about $2 m$ from the camera. The camera was turned man- 
ually around the object, and images are taken every one or two degrees. The first 13 images and the last 11 images have slightly different lighting condition. One gradient image of the sequences is displayed in Figure 1. It is important to note that the images we used are only a small portion, approximatively $1 / 4$ of the original $512 \times 512$ images of the sequence.

- Points are automatically tracked over the sequence and the location of the points are optimized by a nonlinear subpixel corner detector [33]. The automatic tracker may have 2 or 3 points missing for some images, the missing points are added by hand. The 6 points used for experimentation are the visible corners of the two cubic objects, marked by white circles in Figure 1. Concerning the precision of the image points, although corner detection is itself subpixel but the precision of points location can not be considered as subpixel since we can never guarantee that in different images a corner comes from the same physical point in space.

- The dimensions of cubic objects in space are measured by hand to serve as ground truth for comparison with the computed invariants. However these ground truth should be considered relative, since errors in measuring $3 \mathrm{D}$ objects are inevitable. Measurement error was estimated as $\pm 2 \mathrm{~mm}$.

The results in Table VI show that the computed invariants from different triplet of images are stable and usable, except for $\gamma$ in the third row. We note that the computation of $\gamma$ is systematically worse than that of $\alpha$ and $\beta$, this is due to its small modulus, although both have almost the same magnitude of absolute error, about 0.04, the relative error is much more important for $\gamma$ than $\alpha$. This is also due to error accumulation, since $\gamma$ is found using $\alpha$ and $\beta$. One possible remedial measure is to change the order of the computation, or recompute them from the corresponding original cubic equations.

In this table, we also note the influence of the camera distances on the stability of the invariants. Since each pair of neighboring images differs by a rotation of roughly one degree, the camera distance in Table VI can roughly be estimated as the rotation angle in degrees with a radius of two meters. We can see in the table that, globally the results degrade by decreasing the distance between camera positions. However the results from the largest distance do not give the best ones, this can be explained by the fact that one side of the objects in these extreme positions are almost tangent to the view line, so the corresponding corners are not well defined in the images, therefore they can not be precisely located by the corner detector. The pixel errors of the points in the extreme images are much more important than in other images. When the distance between cameras becomes quite small, for instance 2, corresponding to the last 2 rows of the table, the results are considerably degraded, especially for the triplet of the last row. When the camera distance is 1 , the results are too bad to be useful, the results are not presented in the table. Another experiment is performed on a sequence of images of a wooden house. Three views covering about a $45^{\circ}$ ro-

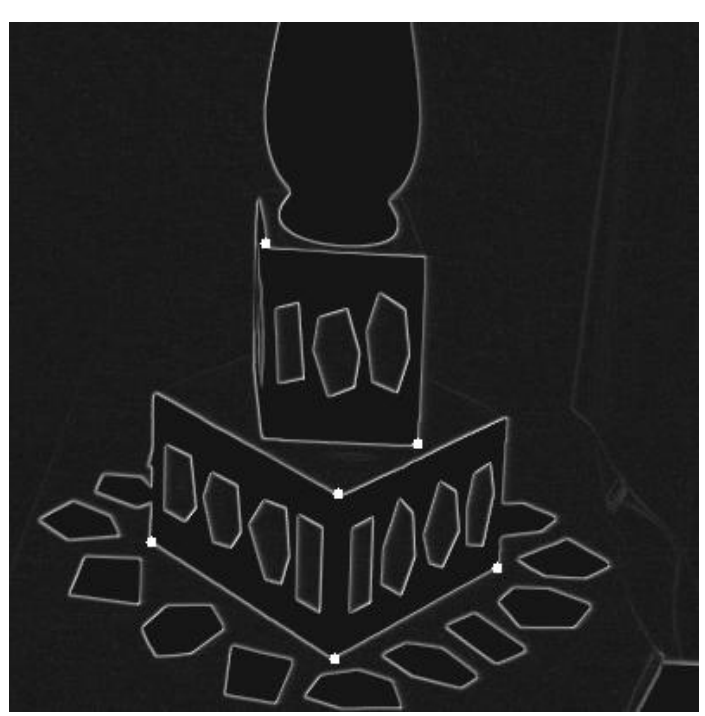

Fig. 1. One gradient image of the sequence. The 6 points marked by a white circle are used for the experiment.

tation of the camera around the wooden house are taken. The corners marked in Figure 2 are tracked for the three images using the same procedure as for the previous image sequence. The 5 reference points are those numbered 2, 5, 8, 10 and 11 in Figure 2. Then with any other point, a set of 6 points is formed. In Table VII, the computed invariants are compared with that computed by transforming the estimated Euclidean coordinates (obtained by the method described in [32]) using the space collineation $A_{4 \times 4}$ ( $c f$. Appendix). In the case of unique real solution, the computed values are followed by a $*$. In case of multiple solutions, the one that is the closest to the known value is selected by hand.

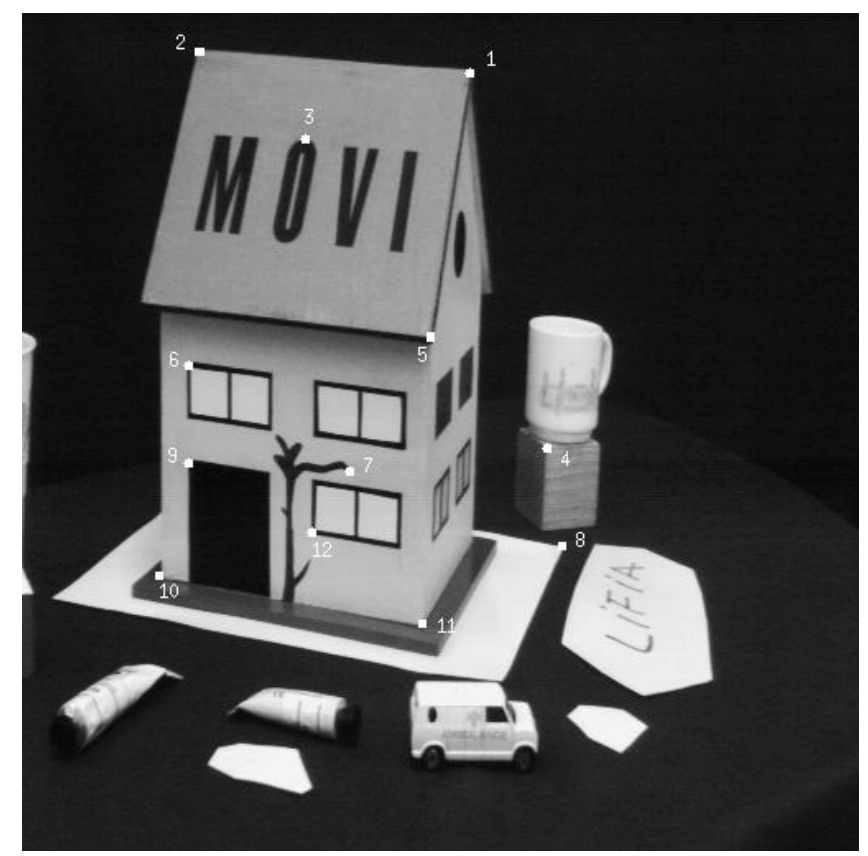

Fig. 2. One of the wooden house image sequence. The points used for the experiment are marked by white circles. 
TABLE of INVARIANTS $\alpha, \beta$ AND $\gamma$ COMPUTED FROM THE REAL IMAGE SEQUENCE WITH DIFFERENT TRIPLETS OF IMAGES. IN THE SECOND COLUMn, camera dist. MEANS THE DISTANCE BETWEEN CAMERA POSITIONS. $d_{i}$ IS THE DIFFERENCE BETWEEN THE COMPUTED VALUE AND THE

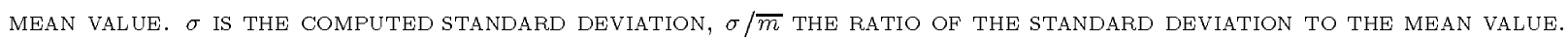

\begin{tabular}{|c||c||c|c||c|c||c|c||}
\hline \hline Ima. triplet & camera dist. & $\alpha$ & $d_{1}$ & $\beta$ & $d_{2}$ & $\gamma$ & $d_{3}$ \\
\hline $1,12,23$ & 12 & $3.055932^{*}$ & -0.032 & $1.322068^{*}$ & -0.014 & $0.044376^{*}$ & -0.014 \\
\hline $2,13,24$ & 12 & 3.040515 & -0.048 & 1.3321695 & -0.0043 & 0.051376 & -0.0065 \\
\hline $1,7,13$ & 5 & 2.981206 & -0.11 & 1.295518 & -0.041 & 0.015540 & -0.0424 \\
\hline $14,19,24$ & 5 & 3.205979 & 0.12 & 1.352756 & 0.016 & 0.056934 & -0.00097 \\
\hline $1,5,9$ & 4 & $3.085201^{*}$ & -0.0032 & $1.336204^{*}$ & -0.00022 & $0.064528^{*}$ & 0.0066 \\
\hline $13,17,21$ & 4 & 3.592866 & 0.50 & 1.370506 & 0.0034 & 0.053793 & -0.0041 \\
\hline $14,18,22$ & 4 & 2.976934 & -0.11 & 1.328929 & -0.0074 & 0.049507 & -0.0084 \\
\hline $2,5,8$ & 3 & $3.006701^{*}$ & -0.082 & $1.359429^{*}$ & 0.023 & $0.117986^{*}$ & 0.060 \\
\hline $3,6,9$ & 3 & $3.111329^{*}$ & 0.023 & $1.353453^{*}$ & 0.017 & $0.084712^{*}$ & 0.027 \\
\hline $14,17,20$ & 3 & 3.417479 & 0.33 & 1.366791 & 0.030 & 0.059527 & 0.0016 \\
\hline $16,19,22$ & 3 & $2.497743^{*}$ & -0.59 & $1.282863^{*}$ & -0.054 & $0.038662^{*}$ & -0.019 \\
\hline \hline $1,3,5$ & 2 & 3.539165 & 0.45 & 1.376995 & 0.041 & 1.140780 & 1.08 \\
\hline $15,17,19$ & 2 & $6.754482^{*}$ & 3.7 & $1.509805^{*}$ & 0.17 & $0.099840^{*}$ & 0.042 \\
\hline \hline mean $\bar{m}$ & - & 3.088353 & - & 1.336426 & - & 0.057904 & - \\
\hline s.d. $\sigma$ & - & 0.28 & - & 0.028 & - & 0.026 & - \\
\hline$\sigma / \bar{m}$ & - & 0.091 & - & 0.021 & - & 0.45 & - \\
\hline ground truth & - & 3.155082 & - & 1.357450 & - & 0.073426 & - \\
\hline \hline
\end{tabular}

TABLE VII

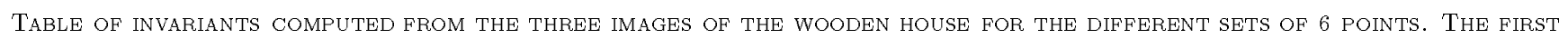
COLUMN PRESENTS THE SETS OF NUMBERS OF 6 POINTS. IN THE SECOND COLUMN, $\alpha^{\prime}, \beta^{\prime}$ AND $\gamma^{\prime}$ ARE TRANSFORMED EUCLIDEAN COORDINATES AS GROUND TRUTH. In the LAST COLUMN, $\alpha, \beta$ AND $\gamma$ ARE COMPUTED FROM THREE IMAGES.

\begin{tabular}{|c|c||c|}
\hline \hline Set of 6 points & known $\left(\alpha^{\prime}, \beta^{\prime}, \gamma^{\prime}\right)$ & computed $(\alpha, \beta, \gamma)$ \\
\hline$\{1,2,5,8,10,11\}$ & $(0.594573,0.944785,0.157327)$ & $\left(0.581565^{*}, 0.925869^{*}, 0.143597^{*}\right)$ \\
\hline$\{2,3,5,8,10,11\}$ & $(0.716795,0.740667,0.64426)$ & $(0.714164,0.739041,0.662835)$ \\
\hline$\{2,4,7,8,10,11\}$ & $(1.68619,12.7413,0.970638)$ & $(1.714522,12.729020,0.995141)$ \\
\hline$\{2,5,6,8,10,11\}$ & $(0.681678,0.241914,1.7126)$ & $\left(0.742775^{*}, 0.325263^{*}, 1.822018^{*}\right)$ \\
\hline$\{2,5,7,8,10,11\}$ & $(-0.22391,0.225495,0.858623)$ & $(-0.230072,0.228533,0.880303)$ \\
\hline$\{2,5,8,9,10,11\}$ & $(0.634079,0.344556,3.09316)$ & $(0.705443,0.448216,3.303431)$ \\
\hline$\{2,5,8,10,11,12\}$ & $(-0.816493,0.264887,2.45476)$ & $(-0.849691,0.233549,2.523767)$ \\
\hline \hline
\end{tabular}

\section{The experiment on projective reconstruction}

As we have seen in Section $\mathrm{V}$ that we can use the invariants to perform projective reconstruction of a point set from 3 uncalibrated images. We experimented on real images of the wooden house (see Figure 2). All tracked 46 points of 3 images are used.

Although the theoretical multiplicity of the projective reconstruction is at most three from the minimal data. With more than the minimum number of points in practice, a unique solution is always obtained. In this experiment, the set of 5 reference points is $\{2,5,8,10,11\}$ (cf. Figure 2). The sixth point used to compute the projection matrices is the point numbered 1 in Figure 2, that is the set of 6 points $\{1,2,5,8,10,11\}$. Since the invariants of this set of points are uniquely determined (see the first row of Table VII), the projection matrices are uniquely computed from these invariants, then any other tracked points are reconstructed from the projection matrices. The projective reconstruction is then transformed into its Euclidean representation (see Fig 3 ) by applying $A_{4 \times 4}^{-1}$ (see Appendix) with the known reference points in order to compare the result with direct Euclidean reconstruction from 5 images [32].

Note that during all reconstruction process, we need to solve only one cubic equation in one variable, all other computational efforts are linear operations.

\section{Conclusion}

In the first part of the paper, it is shown that the three invariants of a set of six points in general position in space can be algebraically computed from three images of these points. No further information about camera calibration and epipolar geometry is needed. It is shown that the maximum number of solutions is three which are all algebraically explicited in closed form.

Compared with the computation of the invariants from two images, which is essentially based on Sturm's method using seven points, the method proposed in this paper needs only six points of the three images.

The result obtained in this paper has some important consequences. It allows to extend the results of Faugeras [1] and Hartley et al. [2] for projective reconstruction and Sturm's method [3] for determining the epipolar geometry from two uncalibrated images to the case of three uncalibrated images with the minimal data of six points instead of seven. It is also shown that a camera could be selfcalibrated with only six points of three images.

In the second part of the paper, the stability of the invari- 
is given by
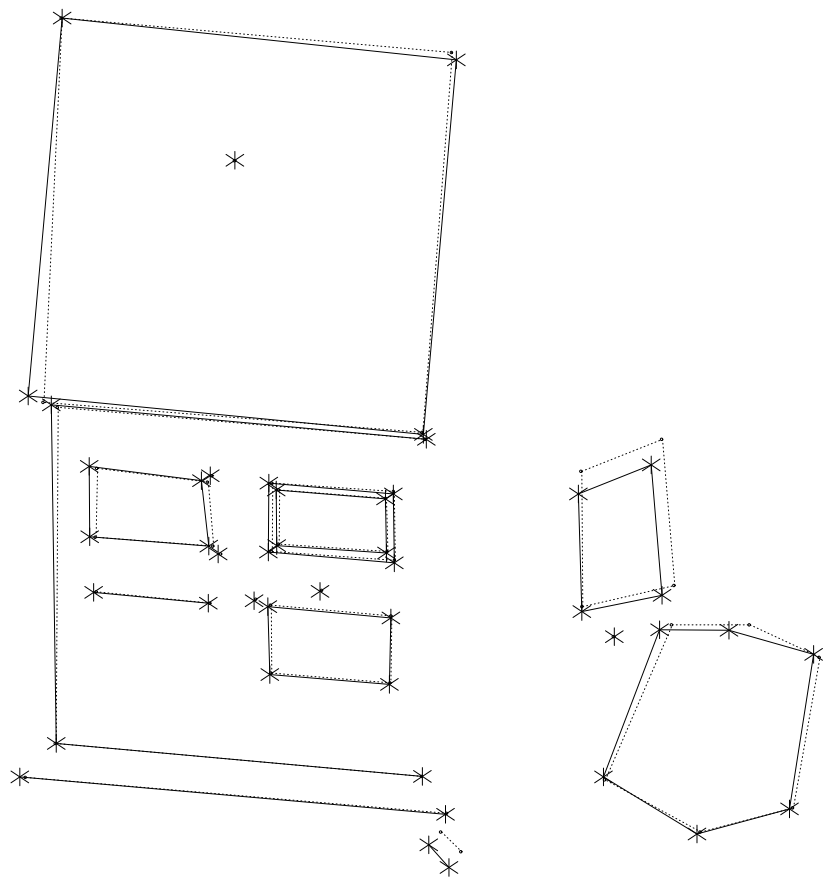

Fig. 3. The rectified projective reconstruction from 3 uncalibrated images: the points are displayed as stars and the line segments as solid lines. Euclidean reconstruction from five images: the points are dots and the line segments dashed lines. The 5 reference points used were $\{2,5,8,10,11\}$ (cf. Fig. 2).

ants is studied via both simulated and real images with respect to different factors. The computed invariants are numerically stable both with respect to pixel noise and to selection of triplet of images if the distance between cameras is not too small. This confirms that our parametrization has a better numerical behavior than Sturm's. We have also successfully applied the method to perform projective reconstruction of set of points.

We can remark that the way we computed the canonical projective coordinates is not symmetric in the six points, it is therefore order-dependent. The study of orderindependent invariants has no theoretical problems, however practically it will produce extremely long and complicated algebraic expression which will compromise numerical stability.

\section{APPENDIX}

\section{Plane collineation $A_{3 \times 3}^{-1}$}

The plane collineation matrix $A_{3 \times 3}^{-1}$, which transforms the canonical projective basis in $\mathcal{P}^{2}$,

$$
\begin{array}{ll}
e_{1}=(1,0,0)^{T}, & e_{2}=(0,1,0)^{T}, \\
e_{3}=(0,0,1)^{T}, & e_{4}=(1,1,1)^{T},
\end{array}
$$

into four given points: $\left\{p_{i}=\left(x_{i}, y_{i}, 1\right)^{T}, i=1, \ldots, 4\right\}$,

$$
e_{i} \rightarrow \mu_{i} p_{i}=A_{3 \times 3}^{-1} e_{i},
$$

$$
\left(\begin{array}{rrr}
x_{1} \Delta_{423} & x_{2} \Delta_{143} & x_{3} \Delta_{124} \\
y_{1} \Delta_{423} & y_{2} \Delta_{143} & y_{3} \Delta_{124} \\
\Delta_{423} & \Delta_{143} & \Delta_{124}
\end{array}\right)
$$

where

$$
\Delta_{i j k}=\operatorname{det}\left(\begin{array}{ccc}
x_{i} & y_{i} & 1 \\
x_{j} & y_{j} & 1 \\
x_{k} & y_{k} & 1
\end{array}\right) .
$$

Space collineation $A_{4 \times 4}^{-1}$

The space collineation matrix $A_{4 \times 4}^{-1}$, which transforms the canonical projective basis,

$$
\begin{array}{ll}
e_{1}=(1,0,0,0)^{T}, & e_{2}=(0,1,0,0)^{T}, \quad e_{3}=(0,0,1,0)^{T}, \\
e_{4}=(0,0,0,1)^{T}, & e_{5}=(1,1,1,1)^{T},
\end{array}
$$

into five given points, $\left\{p_{i}=\left(x_{i}, y_{i}, z_{i}, 1\right)^{T}, i=1, \ldots, 5\right\}$ :

$$
e_{i} \rightarrow \mu_{i} p_{i}=A_{4 \times 4}^{-1} e_{i},
$$

is given by

$$
\left(\begin{array}{rrrr}
x_{1} \Delta_{5234} & x_{2} \Delta_{1534} & x_{3} \Delta_{1254} & x_{4} \Delta_{1235} \\
y_{1} \Delta_{5234} & y_{2} \Delta_{1534} & y_{3} \Delta_{1254} & y_{4} \Delta_{1235} \\
z_{1} \Delta_{5234} & z_{2} \Delta_{1534} & z_{3} \Delta_{1254} & z_{4} \Delta_{1235} \\
\Delta_{5234} & \Delta_{1534} & \Delta_{1254} & \Delta_{1235}
\end{array}\right)
$$

where

$$
\Delta_{i j k l}=\operatorname{det}\left(\begin{array}{cccc}
x_{i} & y_{i} & z_{i} & 1 \\
x_{j} & y_{j} & z_{j} & 1 \\
x_{k} & y_{k} & z_{k} & 1 \\
x_{l} & y_{l} & z_{l} & 1
\end{array}\right) .
$$

Then $A_{3 \times 3}$ and $A_{4 \times 4}$ can then be obtained from its inverse either analytically by computing cofactors or numerically by $L U$ decomposition.

\section{ACKNOWLEDGEMENTS}

This work is partly supported by European Esprit BRA projects Viva which is gratefully acknowledged. We would also like to thank B. Boufama, P. Gros, R. Horaud, R. Mohr, D. Sinclair, D. Wang and C. Zhao for providing interesting discussion and helps.

\section{REFERENCES}

[1] O. Faugeras, "What can be seen in three dimensions with an uncalibrated stereo rig?", in Proceedings of the 2nd European Conference on Computer Vision, Santa Margherita Ligure, Italy, G. Sandini, Ed. May 1992, pp. 563-578, Springer-Verlag.

[2] R. Hartley, R. Gupta, and T. Chang, "Stereo from uncalibrated cameras", in Proceedings of the Conference on Computer Vision and Pattern Recognition, Urbana-Champaign, Illinois, USA, 1992, pp. 761-764.

[3] R. Sturm, "Das Problem der Projektivität und seine Anwendung auf die Flächen zweiten Grades", Math. Ann., vol. 1, pp. 533574,1869

[4] J.L. Mundy and A. Zisserman, Eds., Geometric Invariance in Computer Vision, MIT Press, Cambridge, Massachusetts, USA, 1992 .

[5] J.J. Koenderink and A. J. van Doorn, "Affine structure from motion", Tech. Rep., Utrecht University, Utrecht, The Netherlands, October 1989. 
[6] R. Mohr, L. Quan, F. Veillon, and B. Boufama, "Relative 3D reconstruction using multiples uncalibrated images", Tech. Rep. RT 84-I-IMAG LIFIA 12, LIFIA-IRIMAG, 1992.

[7] R. Mohr, L. Morin, C. Inglebert, and L. Quan, "Geometric solutions to some 3D vision problems", in Integration and Control in Real Time Active Vision, J.L. Crowley, E. Granum, and R. Storer, Eds., EsPRIT BRA Series. Springer-Verlag, 1991.

[8] L. Quan and R. Mohr, "Affine shape representation from motion through reference points", Journal of Mathematical Imaging and Vision, vol. 1, pp. 145-151, 1992, also in IEEE Workshop on Visual Motion, New Jersey, pages 249-254, 1991.

[9] P. Gros and L. Quan, "Projective Invariants for Vision", Tech. Rep. RT 90 IMAG - 15 LIFIA, LifIA-IRIMAG, Grenoble, France, December 1992.

[10] G. Sparr, "An algebraic/analytic method for reconstruction from image correspondance", in Proceedings of the 7th Scandinavian Conference on Image Analysis, Aalborg, Denmark, 1991, pp. $274-281$.

[11] R. Hartley, "Invariants of points seen in multiple images", Tech. Rep., G.E. CRD, Schenectady, 1992.

[12] A. Shashua, "Projective Structure from Two Uncalibrated Images : Structure from Motion and Recognition", Tech. Rep. A.I. Memo No. 1363, Massachusetts Institute of Technology, September 1992 .

[13] D. Forsyth, J.L. Mundy, A. Zisserman, C. Coelho, A. Heller, and C. Rothwell, "Invariant descriptors for 3D object recognition and pose", IEEE Transactions on PAMI, vol. 13, no. 10, pp. 971-991, October 1991.

[14] I. Weiss, "Projective invariants of shapes", in Proceedings of DARPA Image Understanding Workshop, Cambridge, Mas. sachusetts, USA, 1988, pp. 1125-1134.

[15] J.B. Burns, R. Weiss, and E.M. Riseman, "View variation of point set and line segment features", in Proceedings of DARPA Image Understanding Workshop, Pittsburgh, Pennsylvania, USA, 1990, pp. 650-659.

[16] C.A. Rothwell, D.A. Forsyth, A. Zisserman, and J.L. Mundy, "Extracting projective structure from single perspective views of 3D point sets", in Proceedings of the 4th International Conference on Computer Vision, Berlin, Germany, May 1993, pp. 573-582.

[17] E.B. Barrett, M.H. Brill, N.N. Haag, and P.M. Payton, "Invariant linear methods in photogrammetry and model-matching", in Geometric Invariance in Computer Vision, J. Mundy and A. Zisserman, Eds., pp. 277-292. The MIT press, 1992.

[18] P. Gros and L. Quan, "3D projective invariants from two images", in Geometric Methods in Computer Vision II, SPIE's 1993 International Symposium on Optical Instrumentation and Applied Science, July 1993, pp. 75-86.

[19] O.D. Faugeras, Q.T. Luong, and S.J. Maybank, "Camera Self-Calibration: Theory and Experiments", in Proceedings of the 2nd European Conference on Computer Vision, Santa Margherita Ligure, Italy, G. Sandini, Ed. May 1992, pp. 321334, Springer-Verlag.

[20] Q.T. Luong, Matrice Fondamentale et Autocalibration en Vision par Ordinateur, Thèse de doctorat, Université de Paris-Sud, Orsay, France, December 1992.

[21] S.J. Maybank and O.D. Faugeras, "A theory of self calibration of a moving camera", International Journal of Computer Vision, vol. 8, no. 2, pp. 123-151, 1992 .

[22] L. Quan, "Invariants of 6 points from 3 uncalibrated images", in Proceedings of the 3rd European Conference on Computer Vision, Stockholm, Sweden, J.O. Eklundh, Ed. 1994, vol. II, pp. 459-470, Springer-Verlag.

[23] H.C. Longuet-Higgins, "A computer program for reconstructing a scene from two projections", in Nature, vol. 293, pp. 133-135. XX, September 1981 .

[24] A.B. Coble, Algebraic Geometry and Theta Functions, American Mathematical Society, 1961.

[25] J.G. Semple and G.T. Kneebone, Algebraic Projective Geometry, Oxford Science Publication, 1952.

[26] J.H. Grace and A. Young, The Algebra of Invariants, Chelsea Publishing Company, New York, 1903.

[27] J. Dixmier, "Quelques aspects de la théorie des invariants", Gazette des Mathématiciens, vol. 43, pp. 39-64, January 1990.

[28] R. Mohr, F. Veillon, and L. Quan, "Relative 3D reconstruction using multiple uncalibrated images", in Proceedings of the Conference on Computer Vision and Pattern Recognition, New York, USA, June 1993, pp. 543-548. Problems der Homographie von M. Chasles abhängt", J. reine angew. Math., , no. 62, pp. 188-192, 1863.

[30] O. Faugeras and S. Maybank, "Motion from point matches: Multiplicity of solutions", International Journal of Computer Vision, vol. 3, no. 4, pp. 225-246, 1990.

[31] D.C. Brown, "Close-range camera calibration", Photogrammetric Engineering, vol. 37, no. 8, pp. 855-866, 1971.

[32] B. Boufama, R. Mohr, and F. Veillon, "Euclidian constraints for uncalibrated reconstruction", in Proceedings of the 4 th International Conference on Computer Vision, Berlin, Germany, May 1993, pp. 466-470.

[33] R. Deriche and G. Giraudon, "Accurate corner detection: an analytical study", in Proceedings of the 3rd International Conference on Computer Vision, Osaka, Japan, 1990.

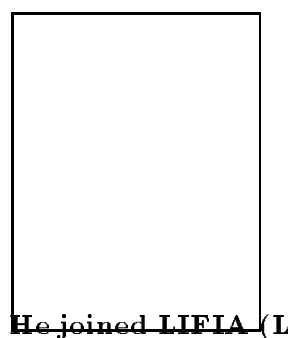

Long Quan was born in TaiYuan, China, on Juin 14, 1964. He received the B.Sc. degree in telecommunication from the Northern Jiao-Tong Univerity, Beijing, China in 1984, the M.Sc. degree in computer science from the University of Nancy $I$ in 1986, and Ph.D. degree in computer science from INPL (Institut National Polytechnique de Lorraine), Nancy, France, in 1989.

Laboratoire d'Informatique Fondamentale et d'Intelligence Artificielle), Grenoble, France, as Research Scientist of CNRS (Centre National de Recherche Scientifique) in 1990. His main research interests are currently on applications of invariant theory and projective geometry for computer vision. 